\title{
An improved exact algorithm and an NP-completeness proof for sparse matrix bipartitioning
}

\author{
Timon E. Knigge Rob H. Bisseling
}

May 8, 2020

\begin{abstract}
We investigate sparse matrix bipartitioning - a problem where we minimize the communication volume in parallel sparse matrix-vector multiplication. We prove, by reduction from graph bisection, that this problem is $\mathcal{N} \mathcal{P}$-complete in the case where each side of the bipartitioning must contain a linear fraction of the nonzeros.

We present an improved exact branch-and-bound algorithm which finds the minimum communication volume for a given matrix and maximum allowed imbalance. The algorithm is based on a maximum-flow bound and a packing bound, which extend previous matching and packing bounds.

We implemented the algorithm in a new program called MP (Matrix Partitioner), which solved 839 matrices from the SuiteSparse collection to optimality, each within 24 hours of CPU-time. Furthermore, MP solved the difficult problem of the matrix cage 6 in about 3 days. The new program is on average more than ten times faster than the previous program MondriaanOpt.

Benchmark results using the set of 839 optimally solved matrices show that combining the medium-grain/iterative refinement methods of the Mondriaan package with the hypergraph bipartitioner of the $\mathrm{PaToH}$ package produces sparse matrix bipartitionings on average within $10 \%$ of the optimal solution.
\end{abstract}

\section{Introduction}

Sparse matrix partitioning is important for the parallel solution of sparse linear systems by direct or iterative methods. In iterative solvers, the basic kernel is the multiplication of a sparse matrix and a dense vector, the SpMV operation. A good partitioning of the sparse matrix and the vector will balance the computation load in a parallel SpMV by spreading the matrix nonzeros evenly over the parts assigned to the processors of the parallel computer, while also leading to less communication of the vector components between the processors. 
In the past decades, much effort has been spent on developing and improving heuristic partitioning methods. In particular, hypergraph methods have been very successful because they model the communication volume (the total number of data words sent) exactly. Two-dimensional (2D) partitioning methods are superior to 1D methods, since they are more general and can split both the rows and columns of the matrix and hence in principle can provide better solutions. Heuristic algorithms for hypergraph-based sparse matrix partitioning have been implemented in the sequential software packages hMetis [1], PaToH [2], Mondriaan [3], KaHyPar [4], and the parallel packages Parkway [5] and Zoltan [6]. The current state-of-the-art methods for 2D sparse matrix partitioning are the fine-grain [7] method and the medium-grain method [8].

How good are the current methods and is it still worthwhile to improve them? To answer this question we need to compare the quality of the outcome, i.e., the communication volume, to the optimal result. To enable such a comparison, we need an exact algorithm that provides the minimum communication volume for a specfied maximum load imbalance. The first exact algorithm for this problem (with two parts) was proposed by Pelt and Bisseling [9], based on a branch-and-bound method. This algorithm has been implemented in the program MondriaanOpt, included in the Mondriaan package, version 4.2. As of today, 356 matrices from the SuiteSparse (formerly University of Florida) sparse matrix collection [10] have been bipartitioned to optimality by MondriaanOpt ${ }^{1}$ Being able to increase the size of the solution subset would be valuable for benchmarking heuristic partitioners, by providing more comparison data of a more realistic size. Heuristic partitioners are aimed at large problems, although they will encounter smaller problems after their inital splits.

Optimal partitionings are easiest to compute for splitting into two parts: the required computation time grows quickly with a larger number of parts, as discussed by Pelt and Bisseling [9]. Furthermore, heuristic partitioners often are based on recursive bipartitioning, so that it is most important to gauge the quality of the bipartitioner. (A notable exception is KaHyPar, which computes a direct $k$-way partitioning.) Therefore, both the exact partitioner implemented in MondriaanOpt and the improved partioner MP (for Matrix Partitioner) presented in this article, compute optimal solutions for bipartitioning. Another question that arises is about the $\mathcal{N} \mathcal{P}$-completeness [11] of the sparse matrix bipartitioning problem. It is known that the decision problem of graph bipartitioning with a tolerated imbalance is $\mathcal{N} \mathcal{P}$-complete [12, Theorem 3.1] and so is hypergraph partitioning [13. Chapter 6], but sparse matrix bipartitioning is a special case of hypergraph bipartitioning (for instance, with vertices contained in only two hyperedges), and whether this problem is $\mathcal{N} \mathcal{P}$-Complete is still open.

The novelty of this paper is twofold: (i) we present an improvement of the previous state-of-the-art exact algorithm [9] by generalizing a matching-based lower bound on the necessary communication to a stronger maximum flow-based bound, and by generalizing a packing bound (using ideas found by Delling et al. ${ }^{1}$ The solutions can be found at http://www.staff.science.uu.nl/ ${ }^{\text {bisse101/Mondriaan/ }}$
Opt/. 
[14]); (ii) we formalize sparse matrix bipartitioning as a decision problem and prove that it is $\mathcal{N} \mathcal{P}$-complete.

The matrix bipartitioning problem that we solve by an exact algorithm can be formulated as follows. Given an $m \times n$ sparse matrix with $|A|$ nonzeros and an allowed imbalance fraction of $\epsilon \geq 0$, find disjoint subsets $A_{1}, A_{2} \subseteq A$ such that

$$
A=A_{1} \cup A_{2},
$$

and

$$
\left|A_{i}\right| \leq(1+\epsilon)\left\lceil\frac{|A|}{2}\right\rceil, \text { for } i=1,2
$$

and such that the communication volume $\operatorname{VOL}\left(A_{1}, A_{2}\right)$ is minimal.

Here, the communication volume is defined as the total number of rows and columns that have nonzeros in both subsets. Each of these cut rows and columns gives rise to one communication of a single scalar in a parallel SpMV. Equation (2) represents a constraint on the load balance of two processors of a parallel computer executing the SpMV.

In this paper, we will only consider the communication volume as the metric to be minimized. Note that other possible objectives, such as minimizing the maximum communication volume per processor or minimizing the total number of messages, may also be relevant. For bipartitioning, however, these metrics need not specifically be optimized: for two processors, the volume per processor is just half the total volume, if we count sending and receiving data as equally important; furthermore, the total number of messages sent is at most two, so there is not much to be optimized. Here, we ignore any costs needed for packing the data words into a message; these costs are proportional to the number of data words to be sent. For partitioning into a larger numbers of parts than two, recursive bipartitioning is often used, and then the history of previous bipartitionings must be taken into account if for instance we also want to minimize the total number of messages. Here, starting up a new message incurs an extra cost, above the cost of sending a data word. This could be done by a sophisticated recursive hypergraph bipartitioning approach [15] that simultaneously reduces the communication volume and the number of messages, thus solving a different optimization problem; this problem, however, is beyond the scope of the present paper.

Many exact partitioning algorithms have been developed for graphs [14, 16, 17, 18, 19]. All these algorithms minimize the edge cut, not the communication volume. Felner 18 solves a graph partitioning problem with uniform edge weights to optimality with a purely combinatorial branch-and-bound method, reaching up to 100 vertices and 1000 edges. Delling et al. [14] solved larger problems using packing-tree bounds and graph contractions, and solved the open street map problem luxembourg with 114,599 vertices and 119,666 edges in less than a minute.

For hypergraphs, much less work has been done on exact partitioning [20, 21, 22]. Kucar 21] uses integer linear programming (ILP) to solve a problem with 1888 vertices, 1920 nets (hyperedges), and 5471 pins (nonzeros) in three days of 
CPU time; the heuristic solver hMetis [1] managed to find a solution in less than a second for the same problem, and it turned out to be optimal. Bisseling and his team members [22] solved an industrial call-graph problem by formulating it as a hypergraph partitioning problem with the cut-net metric, and they solved it heuristically by using Mondriaan and exactly by an ILP method (in 9 days of CPU time).

For exact sparse matrix partitioning, the problem could in principle be formulated as a hypergraph bipartitioning problem by using the fine-grain model [7]: each matrix nonzero becomes a vertex in the hypergraph; the nonzeros in a row are connected by a row-net and the nonzeros in a column by a column-net. Thus, we obtain a hypergraph with $|A|$ vertices and $m+n$ nets, with the special property that each vertex is contained in precisely two nets. One of these nets thus belongs to a group of $m$ pairwise disjoint row-nets, and the other to a group of $n$ pairwise disjoint column-nets. Furthermore, no two vertices have the same pair of nets. An exact general hypergraph partitioner could then be used to solve the problem to optimality. This, however, is less efficient than direct exact sparse matrix partitioning, since the hypergraph partitioner would not exploit the aforementioned properties. In contrast, the direct matrix approach imposes them by construction.

Previous work [9] presented the first direct exact matrix partitioner, implemented in the open-source software MondriaanOpt. This work was optimized and parallelized by Mumcuyan and coworkers [23] who reordered the matrix given to MondriaanOpt (thus changing the order in which the search space was traversed), automatically choosing the best reordering method from a set of methods by a machine-learning approach, and by parallelising the software for a shared-memory computer using OpenMP. Our own improvements, in the present article, are orthogonal to these extensions, so that they can be combined.

The remainder of this paper is organized as follows: Section 2 presents the $\mathcal{N P}$-completeness proof for $\epsilon$-balanced matrix bipartitioning. Section 3 briefly reviews the previously mentioned branch-and-bound algorithm [9] that was implemented in MondriaanOpt, and presents the generalized bounds and their implementation. Section 4 presents the experimental results, comparing MP to MondriaanOpt for 233 small matrices, and giving results for 599 larger matrices that could not be solved by MondriaanOpt within the allotted time. It also presents a comparison between two heuristic methods, $\mathrm{PaToH}$ and Mondriaan, using these optimal partitionings. Section 5 presents the conclusions and discusses possible future work.

\section{Hardness results}

In this section we will formally analyze matrix bipartitioning and prove that it is $\mathcal{N} \mathcal{P}$-Complete, even if we fix the number of processors to $k=2$ (instead of leaving $k \geq 2$ arbitrary). This problem is a special case of the $\mathcal{N} \mathcal{P}$-Complete hypergraph partitioning problem [13, Chapter 6]. 


\section{$2.1 \quad$ Preliminaries}

To begin, let us define a formal decision-variant of the matrix partitioning problem for $k=2$, based on the optimization variant described in Section 1 where the goal is to minimize the total communication volume. We formulate our decision problems in the style of Garey and Johnson [11. Given a fixed $\epsilon \in[0,1)$, we define $\epsilon$-MATRIX BIPARTITION as follows:

\section{$\epsilon$-Matrix Bipartition}

Input: $\quad$ An $m \times n$ matrix $A$, whose nonzeros are precisely indexed by the set $Z \subseteq\{1, \ldots, m\} \times\{1, \ldots, n\}$, and an integer $M$, the required maximum volume.

Question: Does there exist a disjoint partitioning of $Z$ into $Z_{1} \cup Z_{2}$ such that $\left|Z_{i}\right| \leq(1+\epsilon) \frac{|Z|}{2}$ and volume $\operatorname{VOL}\left(Z_{1}, Z_{2}\right) \leq M$ ?

Note that $\epsilon$ is not part of the problem instance, but of the problem definition. Each $\epsilon$ induces its own partitioning problem, and we really have a class of bipartitioning problems here.

Here $\operatorname{VOL}\left(Z_{1}, Z_{2}\right)$ counts the number of rows and columns that have nonze$\operatorname{ros}$ in $Z_{1}$ and $Z_{2}$, as before. Additionally, to simplify presentation we will from here on, without loss of generality, assume that $(1+\epsilon) \frac{|Z|}{2}$ is an integer, rather than place rounding symbols everywhere. Ultimately, all the value of $\epsilon$ does is induce some integer upper bound on the $\left|Z_{i}\right|$. So for any $\epsilon$ we can, for the purposes of whichever problem instance we are considering, replace it with some $\epsilon^{\prime}$ satisfying this integrality constraint, without affecting the bound on $\left|Z_{i}\right|$. Notice that requiring $\left|Z_{i}\right| \leq(1+\epsilon) \frac{|Z|}{2}$ is equivalent to requiring that ||$Z_{1}|-| Z_{2}|| \leq \epsilon|Z|$, and that $\epsilon|Z|$ must be an integer as well.

When thinking about the matrix bipartitioning problem, it is helpful to reformulate it in terms of graphs. Given an $m \times n$ matrix $A$ we can define a bipartite adjacency graph $G(A)=(V(A), E(A))$ with $m$ vertices representing the rows of $A$, and $n$ vertices representing the columns, where a row vertex $r$ and a column vertex $c$ are connected if and only if $A_{r c}$ is nonzero.

This equivalence extends to the partitioning problem. A bipartitioning of the nonzeros of $A$ corresponds to a bipartitioning of the edges of $G(A)$, and the rows and columns contributing to the final volume correspond precisely to the vertices with edges in both sides of the partition. See also Figure 1.
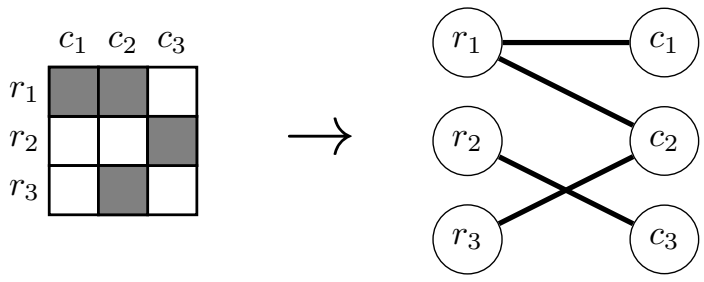

Figure 1: Bipartite graph and matrix equivalence.

This procedure is also reversible, i.e. for any bipartite graph $G$ on $m$ and 
$n$ vertices, we can construct a corresponding matrix $A$ of size $m \times n$ which has nonzeros precisely for the vertices that are connected in $G$. While the nonzero entries of this matrix can have any nonzero value, the associated nonzero pattern is uniquely determined by the edges of $G$.

To this end, we define an equivalent bipartitioning problem on graphs that we will base our reduction on:

\section{$\epsilon$-GRAPH EdGe-Bisection}

Input: $\quad$ Given a graph $G=(V, E)$ and an integer $M$.

Question: Does there exist a disjoint partitioning of $E$ into $E_{1} \cup E_{2}$ such

$$
\text { that }\left|E_{i}\right| \leq(1+\epsilon) \frac{|E|}{2} \text { and }\left|\left(\bigcup_{e \in E_{1}} e\right) \cap\left(\bigcup_{e \in E_{2}} e\right)\right| \leq M ?^{2}
$$

We call a vertex with adjacent edges from both sides of the partitioning 'cut'. The goal is to minimize the number of cut vertices. Additionally, when we explicitly need the partitioning/coloring of $E$, we will write it as a map $\pi: E \rightarrow\{1,2\}$.

\section{$2.2 \epsilon$-Graph Edge-Bisection is $\mathcal{N} \mathcal{P}$-Complete}

We will perform a reduction from the $\epsilon$-GRAPH BISECTION problem, a classical problem first proven $\mathcal{N} \mathcal{P}$-Complete for $\epsilon=0$ [24], and later also for all $\epsilon \epsilon$ $[0,1)[12,25]$.

$$
\begin{array}{ll}
\epsilon \text {-GRAPH BiseCTION } \\
\text { Input: } & \text { A graph } G=(V, E), \text { an integer } M . \\
\text { Question: } & \text { Does there exist a disjoint partitioning of } V \text { into } V_{1} \cup V_{2} \text { with } \\
& \left|V_{i}\right| \leq(1+\epsilon) \frac{|V|}{2} \text { such that }\left|\left\{\{u, v\} \in E \mid u \in V_{1}, v \in V_{2}\right\}\right| \leq \\
& M ?
\end{array}
$$

Analogously to the $\epsilon$-Graph EdGe-Bisection problem, we call an edge with endpoints in both sides of the partitioning 'cut'; the goal is then to minimize the number of cut edges. We similarly write a partitioning of $V$ as a map $\tau: V \rightarrow\{1,2\}$. We can also think of $\tau$ as coloring the vertices in $V$, where one side of the partitioning has the color red, and one side has the color blue. This should not be confused, however, with the classical graph coloring problem, since we allow neighbouring vertices to have the same color.

Let us give a sketch of our proof strategy: given an instance $(G, M)$ of the $\epsilon$-Graph BiseCtion problem, we will build a new graph $G^{\prime}$, whose optimal solution under the $\epsilon$-GRAPH EDGE-BiSECTION problem will give us an optimal solution under $\epsilon$-GRAPH Bisection on $G$.

We first define the construction of such an instance, giving the promised sketch directly after. Let us define the clique expansion of a graph. A visual example (with smaller $S$, for legibility) is also given in Figure 2

Definition 2.1. Given a graph $G=(V, E)$, let $S=4+2|V|\left(\begin{array}{c}|E| \\ 2\end{array}\right)$. We define the clique expansion $\mathcal{K}(G)=\left(V^{\prime}, E^{\prime}\right)$ as first taking a disjoint union of $|V|$ copies

\footnotetext{
${ }^{2}$ If we write an edge as the set $\{u, v\} \subset V$, then $\bigcup_{e \in E_{1}} e$ gives precisely the set of vertices touched by $E_{1}$.
} 
of the complete graph $K_{S}$. Then, labelling the edges in $E$ as $e_{1}, e_{2}, \ldots, e_{|E|}$, for each edge $e_{i}=\{u, v\}$ we merge the $i^{\text {th }}$ vertex of the clique $K_{S}$ representing $u$ with the $i^{\text {th }}$ vertex of the clique $K_{S}$ representing $v$.

Here, the chosen clique size $S$ will allow us to prove several useful propositions later. Notice that by construction, each pair of cliques is merged at most once, each time in a previously unmerged vertex. As a consequence, while two cliques can share a single vertex, they do not share edges.

Informally, for each vertex $u \in V$ we create a clique $K_{u}$ of size $S$. For every edge $\{u, v\} \in E$, we merge two vertices in the cliques $K_{u}$ and $K_{v}$ together into a single vertex. We then solve the $\epsilon$-GRAPH EDGE-Bisection problem on the resulting graph $\left(V^{\prime}, E^{\prime}\right)$, and translate the resulting coloring of its edges into a coloring of the vertices of $(V, E)$. (Here, the colors correspond to the two parts in the partition.) We note that if each clique in $\left(V^{\prime}, E^{\prime}\right)$ is colored monochromatically then we can color each vertex in $(V, E)$ with the color of its corresponding clique in $\left(V^{\prime}, E^{\prime}\right)$. Then an edge between two differently colored vertices in $(V, E)$ will correspond precisely with a vertex shared by two differently colored cliques in $\left(V^{\prime}, E^{\prime}\right)$. The $\epsilon$-GRAPH EdGE-Bisection problem gives us no guarantee that each clique is indeed colored monochromatically, but we will work around this later.
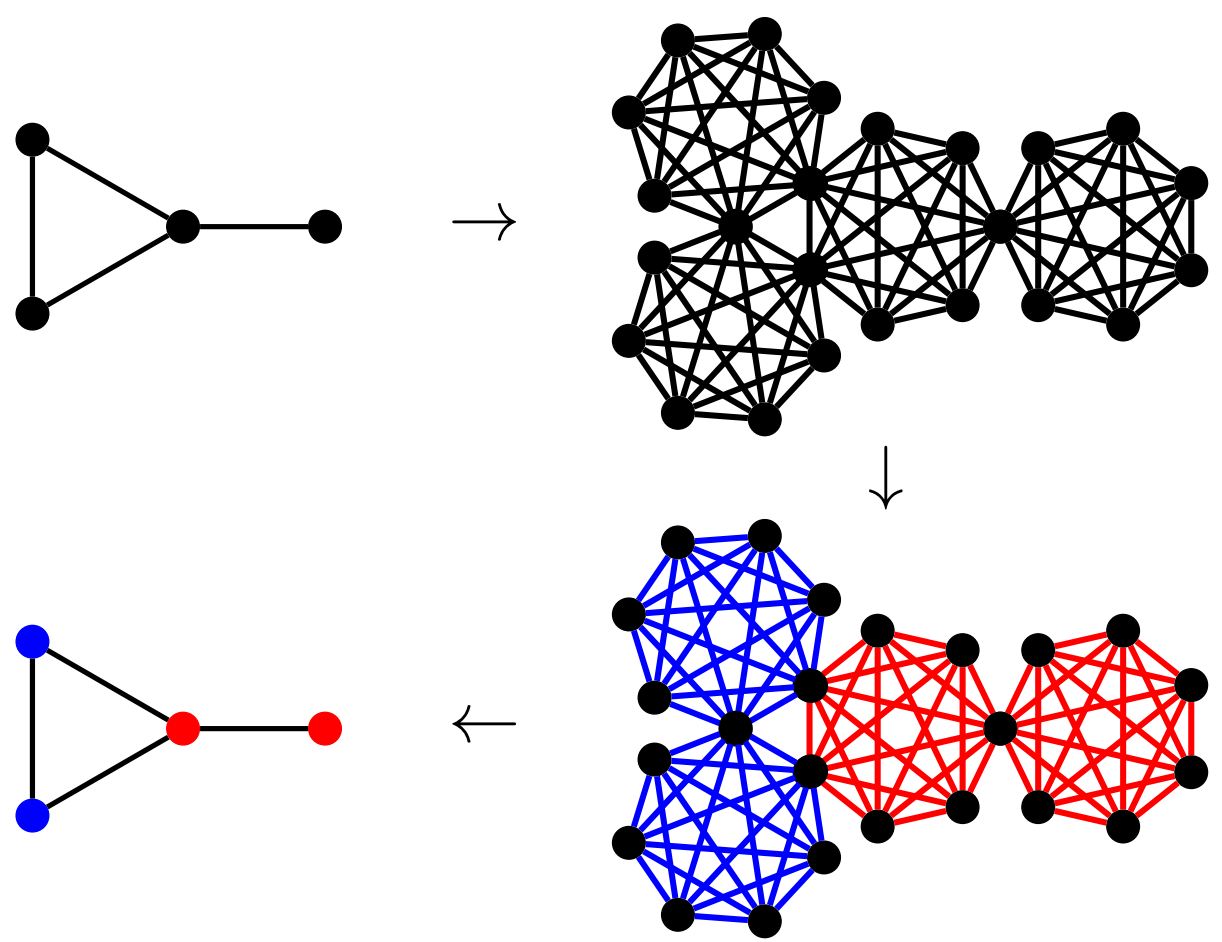

Figure 2: Solving $\epsilon$-Graph Bisection using $\epsilon$-Graph Edge-Bisection . 
Throughout this section we will make a slight abuse of terminology. A clique usually (and up until now) refers to any collection of pairwise connected vertices. However, from now on, when we talk about 'cliques' in $\mathcal{K}(G)$ we will be referring specifically to the cliques corresponding to vertices, i.e. the cliques $\left\{K_{u} \mid u \in V\right\}$ in $\mathcal{K}(G)$.

So after building $\mathcal{K}(G)$ from the $\epsilon$-GRAPH BIsECTION instance $G$, we can solve the $\epsilon$-Graph EdGE-Bisection problem on it. We now want to show that both problems have optimal solutions of equal volume (cost). For a graph $G=(V, E)$, let $\operatorname{GB}_{\epsilon}(G)$ denote the volume of any optimal solution of the $\epsilon$ Graph Bisection problem on $G$, and let $\operatorname{GEB}_{\epsilon}(\mathcal{K}(G))$ denote the volume of any optimal solution of the $\epsilon$-GRAPH EDGE-BISECTION problem on its clique expansion $\mathcal{K}(G)$.

Proposition 2.2. For any graph $G$ we have $\operatorname{GEB}_{\epsilon}(\mathcal{K}(G)) \leq \mathrm{GB}_{\epsilon}(G)$.

Proof. Consider any valid $\epsilon$-balanced bipartitioning $\tau$ of $G=(V, E)$ of cost $M$ (that is, there are exactly $M$ edges $\{u, v\}$ with $\tau(u) \neq \tau(v)$ ). For any vertex $u$, color all edges in the corresponding clique $K_{u}$ in $\mathcal{K}(G)=\left(V^{\prime}, E^{\prime}\right)$ with the same color, i.e. for any edge $e$ in $K_{u}$ let $\pi(e):=\tau(u)$, giving a partitioning $E^{\prime}=E_{1}^{\prime} \cup E_{2}^{\prime}$.

Since each clique $K_{u}$ in $\mathcal{K}(G)$ has the same number of edges, $\left(\begin{array}{l}S \\ 2\end{array}\right)$, and by assumption the partitioning of $V$ satisfies $\left|V_{i}\right| \leq(1+\epsilon) \frac{|V|}{2}$, it follows that $\left|E_{i}^{\prime}\right| \leq(1+\epsilon) \frac{|V|}{2}\left(\begin{array}{c}S \\ 2\end{array}\right)=(1+\epsilon) \frac{\left|E^{\prime}\right|}{2}$.

Now let $s$ be a vertex in $\mathcal{K}(G)$. If $s$ is contained in only one clique, it cannot be cut, since we color the edges of each $K_{u}$ monochromatically. If $s$ is shared by two cliques $K_{u}$ and $K_{v}$, then $s$ corresponds to the edge $e=\{u, v\} \in E$, and we can see that this vertex is cut by $\pi$ if and only if $e$ is cut by $\tau$ (since $K_{u}$ and $K_{v}$ are colored like $u$ and $v$ respectively). Since by construction, each vertex is in at most two cliques, there is no ambiguity.

So the number of cut vertices in the induced partitioning $\pi$ of the edges of $\mathcal{K}(G)$ is exactly the number of cut edges in the original partitioning $\tau$ of the vertices of $G$. We can then minimize over all valid partitionings $\tau$ of $G$ to achieve the desired inequality.

Unfortunately, the converse is harder to prove since we cannot guarantee that an optimal partitioning of $\mathcal{K}(G)$ colors each clique monochromatically. It turns out however, that we can still deterministically associate a color with each clique, provided we have an optimal partitioning.

Definition 2.3. Let $K$ be a clique and suppose we have a coloring of its edges. The dominating color of $K$ is a color $c$ such that there exists a vertex in $K$ with all of its adjacent edges colored $c$.

While this property is not well-defined in general, it is for our restricted case:

Lemma 2.4. Given a graph $G=(V, E)$ and an optimal partitioning $\pi$ of the edges of its clique expansion $\mathcal{K}(G)$. Then each clique $K_{u}$ in $\mathcal{K}(G)$ has a welldefined dominating color. 
Proof. Fix a clique $K_{u}$ in $\mathcal{K}(G)$. We need to prove existence and uniqueness of its dominating color.

First we prove uniqueness: to the contrary, assume there are two vertices $r, b$ in $K_{u}$ such that $r$ has only red edges adjacent, and $b$ only blue edges. Since $K_{u}$ is a clique, the edge $\{r, b\}$ exists, which must be both red and blue, a contradiction.

As for existence, assume to the contrary that every vertex in $K_{u}$ has both blue and red edges adjacent. But this means each vertex in $K_{u}$ is cut by the partitioning $\pi$, and so the cost of this partitioning of $\mathcal{K}(G)$ is at least the clique size $S=4+2|V|\left(\begin{array}{c}|E| \\ 2\end{array}\right)$. One may verify that for any graph, $S>|E|$. However, $|E|$ is a trivial upper bound on the $\epsilon$-Graph BisECTION problem on $(V, E)$ (in which we cut every edge in $E$ ), which, by Proposition 2.2 is an upper bound on the optimal partitioning of the edges of $\mathcal{K}(G)$. Since we assumed our partitioning $\pi$ is optimal, i.e. has cut size exactly equal to $\operatorname{GEB}_{\epsilon}(\mathcal{K}(G))$, this implies that

$$
|E| \geq \operatorname{GB}_{\epsilon}(G) \geq \operatorname{GEB}_{\epsilon}(\mathcal{K}(G)) \geq S>|E|
$$

which is a contradiction, so there must exist a vertex that only has adjacent edges of a single color.

In addition to the above, we would like to note in particular that by definition, if $K_{u}$ has dominating color $c$ (meaning there is a vertex $u^{\prime}$ with all adjacent edges colored $c$ ), then any vertex in $K_{u}$ has at least one adjacent edge with color $c$ (namely the one connecting it to $u^{\prime}$ ).

We now have the tools to formulate a proof strategy: we will color vertices in $G$ by the dominating color of their cliques in an optimal partitioning of $\mathcal{K}(G)$.

Proposition 2.5. For any graph $G$ we have $\operatorname{GB}_{\epsilon}(G) \leq \operatorname{GEB}_{\epsilon}(\mathcal{K}(G))$.

Proof. Fix any optimal partitioning $\pi$ of $\mathcal{K}(G)=\left(V^{\prime}, E^{\prime}\right)$, and let $\tau$ color each vertex $u$ in $G=(V, E)$ with the dominating color of its associated clique $K_{u}$ in $\mathcal{K}(G)$. We would like to prove two things about this partitioning $\tau$ : that the number of cut edges in $G$ is no more than the number of cut vertices in $\mathcal{K}(G)$, and that it is a balanced partitioning of $V$.

We first show the number of edges cut by $\tau$ in $G$ is at most the number of vertices cut by $\pi$ in $\mathcal{K}(G)$. Suppose $\tau$ cuts edge $e_{i}=\{u, v\} \in E$, that is, $\tau(u) \neq \tau(v)$. This means that the dominating colors of $K_{u}$ and $K_{v}$ are different, say without loss of generality that $K_{u}$ is red and $K_{v}$ is blue. Hence, the vertex $s$ in $\mathcal{K}(G)$ that corresponds to $e_{i}$, which we obtained during construction by merging the $i^{\text {th }}$ vertex of $K_{u}$ with the $i^{\text {th }}$ vertex of $K_{v}$, must have red edges adjacent, because it is contained in $K_{u}$, and blue edges, because it is contained in $K_{v}$. So $\pi$ cuts $s$. Since for every edge $e_{j} \in E$ we merged different vertices (specifically, for $e_{j}$ we used the $j^{t h}$ vertex of the two cliques), each edge in $E$ cut by $\tau$ has a unique corresponding vertex $s$ in $\mathcal{K}(G)$ cut by $\pi$, proving the first part.

Next, to show that $\tau$ is a balanced partitioning of $V$, we will equivalently show that our optimal partitioning $\pi$ of $\mathcal{K}(G)$ colors no more than $(1+\epsilon) \frac{|V|}{2}$ cliques with red as their dominating color, and the same for blue. 
Let $r, b \geq 0, r+b=|V|$, count these quantities, assuming without loss of generality that $r \geq b$. First we derive a lower bound on the number of red edges in a clique in $\mathcal{K}(G)$. In each red clique we have at most $|E|$ cut vertices (since we assumed our partitioning was optimal, as in the proof of Lemma 2.4 in fact, across all cliques there are at most $|E|$ cut vertices, but for a lower bound this will suffice), and the edges between two such vertices may be blue, but none of the other $S-|E|$ vertices in this clique are cut, so all other edges should be red, and a lower bound on the number of red edges in $\pi$ is

$$
r\left(\left(\begin{array}{c}
S \\
2
\end{array}\right)-\left(\begin{array}{c}
|E| \\
2
\end{array}\right)\right) \text {. }
$$

Similarly, we can find an upper bound for the number of blue edges by the following reasoning: we color each blue-dominated clique entirely blue, and as many edges as possible in each red-dominated clique (at most $\left(\begin{array}{c}|E| E \mid \\ 2\end{array}\right)$, as before). This gives as an upper bound

$$
b\left(\begin{array}{c}
S \\
2
\end{array}\right)+r\left(\begin{array}{c}
|E| \\
2
\end{array}\right) .
$$

But since $\pi$ was an optimal balanced partitioning of the edges of $\mathcal{K}(G)$, certainly the lower bound on the number of red edges must be smaller than or equal to the upper bound on the number of blue edges, plus the imbalance allowed by $\epsilon$ :

$$
r\left(\left(\begin{array}{c}
S \\
2
\end{array}\right)-\left(\begin{array}{c}
|E| \\
2
\end{array}\right)\right)-\left(b\left(\begin{array}{c}
S \\
2
\end{array}\right)+r\left(\begin{array}{c}
|E| \\
2
\end{array}\right)\right) \leq \epsilon\left|E^{\prime}\right|=\epsilon|V|\left(\begin{array}{c}
S \\
2
\end{array}\right) .
$$

Reordering terms gives:

$$
(r-b-\epsilon|V|)\left(\begin{array}{c}
S \\
2
\end{array}\right) \leq 2 r\left(\begin{array}{c}
|E| \\
2
\end{array}\right) .
$$

Recall that we took $S=4+2|V|\left(\begin{array}{c}|E| \\ 2\end{array}\right)$. Since $S \geq 4$ we have $S \leq\left(\begin{array}{c}S \\ 2\end{array}\right)$, as well as $r \leq|V|$. So if Equation (3) holds, then certainly the following holds:

$$
(r-b-\epsilon|V|) S \leq 2|V|\left(\begin{array}{c}
|E| \\
2
\end{array}\right) .
$$

Substituting $S$ and rewriting we get

$$
4(r-b-\epsilon|V|)+2(r-b-1-\epsilon|V|)|V|\left(\begin{array}{c}
|E| \\
2
\end{array}\right) \leq 0 .
$$

Since we assumed $\epsilon|V| \in \mathbb{N}$ we also have $(r-b-\epsilon|V|) \in \mathbb{Z}$. Then the inequality can only be true if $r-b-\epsilon|V| \leq 0$, and since we assumed $r \geq b$, this gives

$$
0 \leq r-b \leq \epsilon|V|,
$$

hence our partitioning is balanced in $G=(V, E)$. 
Combining the obtained results, we can turn any optimal solution to the $\epsilon$-Graph Edge-Bisection problem on $\mathcal{K}(G)$ into a solution of equal value to the $\epsilon$-Graph Bisection problem on $G$, proving Proposition 2.5.

We are now almost ready to show that $\epsilon$-GRAPH EdGE-Bisection is $\mathcal{N} \mathcal{P}$ Complete. All that remains is showing that it is in $\mathcal{N} \mathcal{P}$ :

Proposition 2.6. The size of $\mathcal{K}(G)=\left(V^{\prime}, E^{\prime}\right)$ is polynomial in the size of $G=(V, E)$.

Proof. By construction, each vertex in $V$ induces a subgraph with $O\left(|V||E|^{2}\right)$ vertices and $O\left(|V|^{2}|E|^{4}\right)$ edges. After merging, the graph will only become smaller. So $\left|V^{\prime}\right|$ is $O\left(|V|^{2}|E|^{2}\right)$ and $\left|E^{\prime}\right|$ is $O\left(|V|^{3}|E|^{4}\right)$.

We can now conclude:

Theorem 2.7. $\epsilon$-Graph Edge-Bisection is $\mathcal{N} \mathcal{P}$-Complete.

Proof. We claim $\epsilon$-Graph Bisection $\leq_{\mathcal{P}} \epsilon$-Graph Edge-Bisection. For a given instance of $\epsilon$-Graph Bisection $(G, M)$, by Proposition 2.2 and Proposition 2.5 we know $\operatorname{GB}_{\epsilon}(G) \leq M$ if and only if $\operatorname{GEB}_{\epsilon}(\mathcal{K}(G)) \leq M$. So if we can solve $\epsilon$-Graph EdGe-Bisection on $\mathcal{K}(G)$ (which has size polynomial in the size of $G$, by Proposition 2.6 in polynomial time, we can also solve $\epsilon$-GraPH Bisection on $G$ in polynomial time.

\section{$2.3 \quad \epsilon$-MATRiX Bipartition is $\mathcal{N} \mathcal{P}$-Complete}

We now consider the original $\epsilon$-Matrix Bipartition problem. As mentioned in Subsection 2.1, it is equivalent to partitioning the edges of a graph. We would like to immediately draw an equivalence between $\epsilon$-MATrix Bipartition and $\epsilon$-GrAPH EdGe-BISECTION - but note that for a matrix $A$ the associated graph $G(A)$ is always bipartite. Therefore, not every graph on which we might want to solve the $\epsilon$-GRAPH EDGE-BISECTION problem can be immediately converted to an equivalent matrix in the context of the $\epsilon$-MATRIX BIPARTITION problem. However, we can resolve this:

Definition 2.8. Given a graph $G=(V, E)$, its edge-split graph $\mathcal{S}(G)=\left(V^{\prime}, E^{\prime}\right)$ is given as:

$$
\begin{gathered}
V^{\prime}=V \cup\left\{v_{e} \mid e \in E\right\} \\
E^{\prime}=\bigcup_{e=\{u, w\} \in E}\left\{\left\{u, v_{e}\right\},\left\{v_{e}, w\right\}\right\} .
\end{gathered}
$$

In other words, we replace each edge by a path of length two. The resulting graph is bipartite (with sides $V$ and $V^{\prime} \backslash V$ ). Using this bipartite extension of a graph, we can build a matrix and use $\epsilon$-MATRIX BIPARTITION to solve the $\epsilon$-Graph EdGe-BiseCtion problem.

First, we prove that we can safely take the edge-split graph without affecting the $\epsilon$-Graph EdGe-Bisection problem. 
Proposition 2.9. For any graph $G$, we have $\operatorname{GEB}_{\epsilon}(G)=\operatorname{GEB}_{\epsilon}(\mathcal{S}(G))$.

Proof. Let $G=(V, E)$ and $\mathcal{S}(G)=\left(V^{\prime}, E^{\prime}\right)$.

$(\geq)$ Let $\pi$ be an optimal coloring of $E$. We define a coloring $\pi^{\prime}$ of $E^{\prime}$ as follows. Let $e^{\prime}=\left\{u, v_{e}\right\} \in E^{\prime}$ with $u \in V \subseteq V^{\prime}$ and $v_{e} \in V^{\prime} \backslash V$. So $e^{\prime}$ is half of the length-two path associated with $e$ in $G$. We set $\pi^{\prime}\left(e^{\prime}\right)=\pi(e)$, that is, we give each edge in $E^{\prime}$ the color of the edge in $E$ that induced it.

Then no vertices in $V^{\prime} \backslash V$ were cut, since both edges in a path have the same color, and the vertices cut by $\pi^{\prime}$ in $V \subseteq V^{\prime}$ are precisely those cut by $\pi$ in $V$.

$(\leq)$ Let $\pi^{\prime}$ be any optimal coloring of $E^{\prime}$. Now for each edge $e \in E$ there are three possibilities:

- Both corresponding edges in $E^{\prime}$ are colored red by $\pi^{\prime}$.

- Both corresponding edges in $E^{\prime}$ are colored blue by $\pi^{\prime}$.

- The corresponding edges in $E^{\prime}$ are colored with two colors.

Let these quantities be counted by $n_{r}, n_{b}$ and $n_{r b}$, respectively. Then $|E|=n_{r}+n_{b}+n_{r b}$. Also the number of red and blue edges in $\pi^{\prime}$ is counted by $2 n_{r}+n_{r b}$ and $2 n_{b}+n_{r b}$, respectively. But by the balancing constraint we get

$$
\left|\left(2 n_{r}+n_{r b}\right)-\left(2 n_{b}+n_{r b}\right)\right| \leq \epsilon\left|E^{\prime}\right|=2 \epsilon|E| .
$$

From this we can derive that $\left|n_{r}-n_{b}\right| \leq \epsilon|E|$. That is, if we have an edge in $G$ whose associated pair in $\mathcal{S}(G)$ is monochrome, then copying these colors back into a coloring (for now ignoring those edges in $G$ whose associated pair is not monochrome) gives a coloring that already satisfies the load balancing constraint. This induced partial coloring will form the basis of our optimal coloring of $E$.

What remains is to assign the bicolored pairs a color. We claim that we can recolor such a pair to a single color without increasing the volume of the solution. Indeed suppose without loss of generality that $e=\{u, w\} \in E$ satisfies $\pi^{\prime}\left(\left\{u, v_{e}\right\}\right)=1$ and $\pi^{\prime}\left(\left\{v_{e}, w\right\}\right)=2$. Now construct a coloring $\pi^{\prime \prime}$ identical to $\pi^{\prime}$ except that $\pi^{\prime \prime}\left(\left\{v_{e}, w\right\}\right)=1$.

So $\pi^{\prime}$ and $\pi^{\prime \prime}$ are identical on the edges adjacent to $V^{\prime} \backslash\left\{v_{e}, w\right\}$, and hence cut the same vertices here. Note that $\pi^{\prime}$ also cuts $v_{e}$, but $\pi^{\prime \prime}$ does not. So even if $w$ now goes from 'not cut' to 'cut', this does not matter since 'uncutting' $v_{e}$ compensates for this.

Applying a change like this decreases $n_{r b}$ by one, and increases $n_{r}$ or $n_{b}$ by one (depending on the choice of color), therefore changing $\left|n_{r}-n_{b}\right|$ by one. Let us now distinguish two cases: 
$(\epsilon|E| \geq 1)$ Since a priori we have $\left|n_{r}-n_{b}\right| \leq \epsilon|E|$, we can repeatedly either increase $n_{r}$ or $n_{b}$ by one while never violating the balancing constraint.

$(\epsilon|E|=0)$ In this case we must in fact have

$$
2 n_{r}+n_{r b}=2 n_{b}+n_{r b}
$$

and hence $n_{r}=n_{b}$. Since $|E|$ is even (otherwise no partitioning exists), we get that $n_{r b}=|E|-\left(n_{r}+n_{b}\right)$ is even as well. Therefore we can pair up such bicolored pairs, making one of them fully red and one fully blue at each step, causing no change in the value of $\left|n_{r}-n_{b}\right|$.

In either situation we can find a new coloring $\pi^{\prime \prime}$ of $E^{\prime}$ with equal volume but with no bicolored pairs. Additionally, the number of monochrome pairs of each color satisfies the load balancing constraint $\left|n_{r}-n_{b}\right| \leq \epsilon|E|$ of the original graph. So we can then map this coloring to $E$, like in case $(\geq)$.
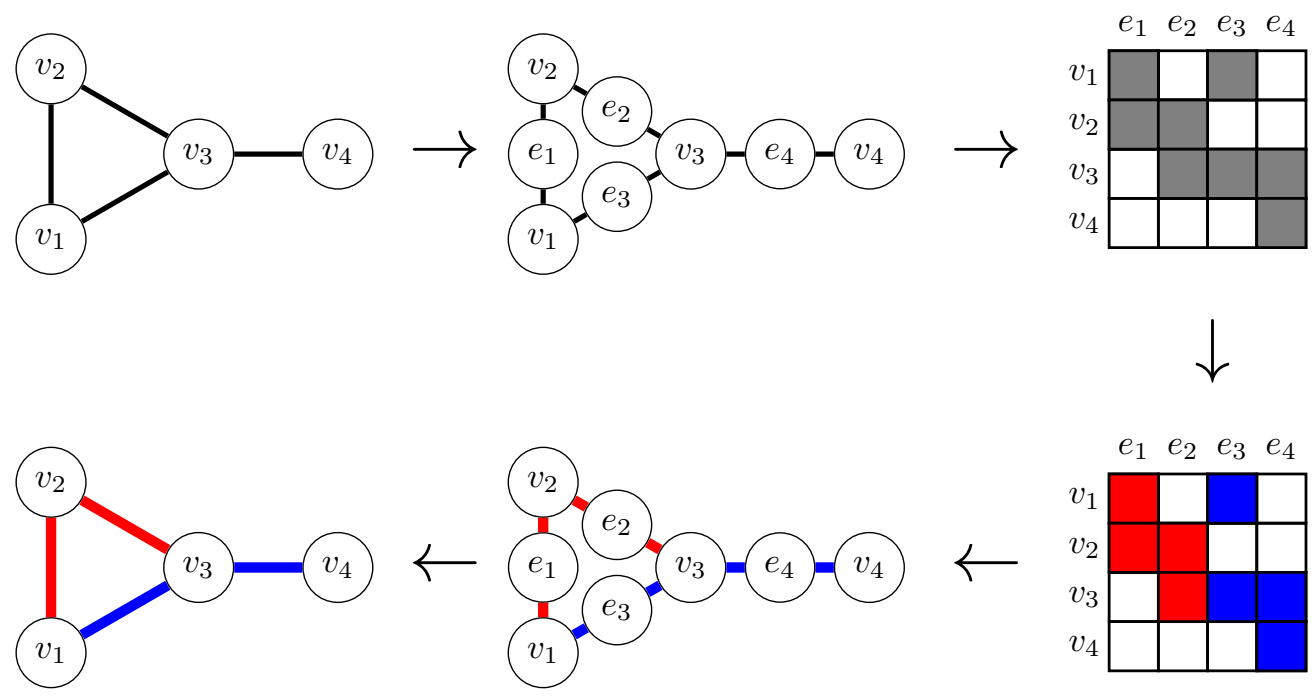

Figure 3: Solving $\epsilon$-Graph Edge-Bisection using $\epsilon$-Matrix Bipartition .

Now we can turn any graph into a bipartite graph without changing its smallest edge bisection. Using this we can prove the main theorem of this section:

Theorem 2.10. $\epsilon$-MATRIX BIPARTITION is $\mathcal{N} \mathcal{P}$-Complete. 
Proof. We will show that

$\epsilon$-Graph Edge-Bisection $\leq_{\mathcal{P}} \epsilon$-Matrix Bipartition

Given a graph $G$, let $G^{\prime}=\mathcal{S}(G)=\left(L \cup R, E^{\prime}\right)$. Create a $|L| \times|R|$ matrix $A$ with $A_{i j}=1$ if $i \in L$ and $j \in R$ are connected by an edge from $E^{\prime}$, and 0 otherwise.

We can now solve the $\epsilon$-Matrix Bipartition problem on $A$, and using the correspondence between matrices and bipartite graphs described in Subsection 2.1. we can turn this into an $\epsilon$-balanced partitioning of $E^{\prime}$, since we have a correspondence between the partitioning of edges and the partitioning of nonzeros, and a correspondence between cutting vertices and cutting rows and columns. This is displayed in Figure 3.

Now Proposition 2.9 and its proof give us a constructive algorithm to transition between $G^{\prime}$ and $G$, solving $\epsilon$-Graph Edge-Bisection on $G$.

\section{Exact Algorithm}

In this section we give an exact algorithm for finding an optimal $\epsilon$-balanced bipartitioning of a matrix, extending the branch-and-bound algorithm by Pelt and Bisseling [9]. They approach the problem with a branch-and-bound technique and use combinatorial bounds to bound the volume of a partial partitioning from below. A major drawback of their bounds however, is that they are in some sense 'local'. They only consider the direct neighbourhoods of assigned sections of the matrix (or rather, its underlying graph). We extend both bounds to the entire graph to take full advantages of its connectivity. We will first briefly discuss the branch-and-bound algorithm, before looking at the two classes of bounds.

\subsection{Branch and Bound}

Recall that a branch-and-bound algorithm initially starts with (a representation of) the whole solution space, and then repeatedly branches on properties of the solutions until these are refined enough that they specify a single solution (this is a leaf in the branch-and-bound tree). When the properties are chosen carefully, we may prune ('bound') large parts of the search tree well before we reach a leaf.

In the case of matrix bipartitioning, a first obvious choice would be to branch on which partition to put each nonzero in. For an $m \times n$ matrix with $N$ nonzeros this results in $2^{N}$ leaf nodes. However, this is not our only option. Instead, we can branch on the status of each of the rows and columns of the matrix: each of them is either entirely red, entirely blue, or 'cut', i.e. it contains both colors. As a result, we only have $3^{m+n}$ leaves, which is already smaller than $2^{N}$ when $m+n<\log _{3}(2) N \approx 0.63 N$. In fact, not all of the $3^{m+n}$ states are even reachable: if a row and column intersect in a nonzero, we cannot mark one of them as red and one of them as blue (i.e., we do require assignments to be consistent). 
When we traverse the branch-and-bound tree, at each stage we have a 'partial assignment', where some of the rows and columns are red, blue, or cut, and some are still unassigned. For a given matrix $A$ and its bipartite graph representation $G(A)=(V, E)$ (recall the equivalence from Section 2), we will write $R \subseteq V$ (resp. $B, C \subseteq V$ ) for the vertices (corresponding to rows and columns) that were assigned red (resp. blue, cut). Additionally, while all remaining vertices are unassigned, they may still be connected to vertices in $R, B$ and $C$. For example, if an unassigned column vertex $u$ is adjacent to a row vertex $r \in R$, this means that $A_{r u}$ is nonzero. In particular, since row $r$ is red, we cannot make $u$ blue. So we will call $u$ partially red, with the corresponding subset of $V$ written as $P_{R}$ (with $P_{B}$ defined analogously). Finally, an unassigned vertex may have neighbours in both $R$ and $B$. Although it is unassigned, we are forced to cut this vertex. Because of this, we assume that whenever such a vertex is created, we immediately cut it. Therefore we will ignore these vertices.

Now we need to find a lower bound for any extension to a partial partition. First we can take $|C|$ as our starting point. We then turn to the unassigned region of the bipartite graph (matrix) to derive stronger bounds.

\subsection{Flow Bounds}
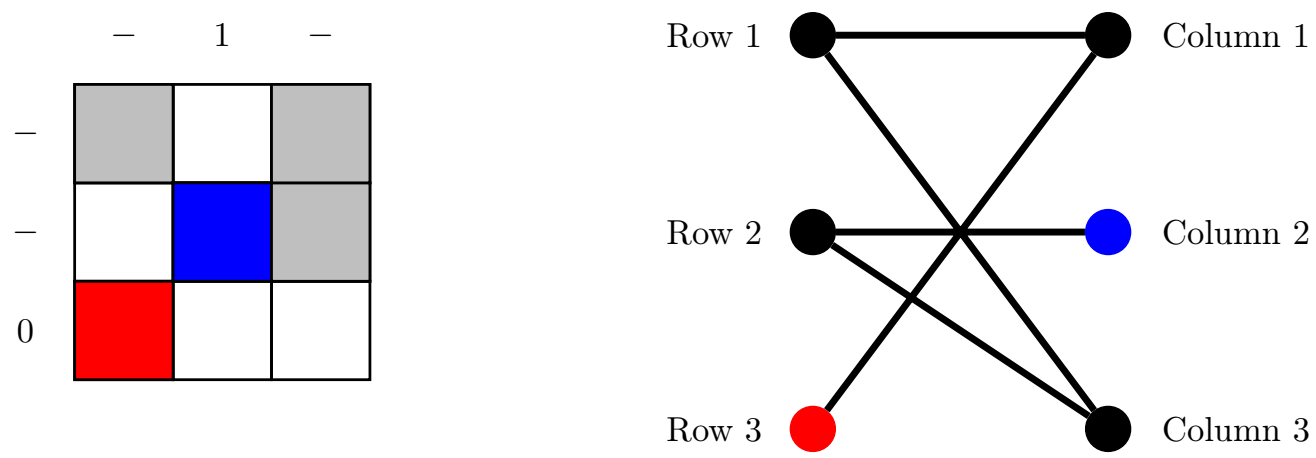

Figure 4: Looking at the matrix, it is not a priori clear that row 3 and column 2 are connected. In the graph representation of the matrix we can clearly see the connecting path.

The first way to find a lower bound on a given partial partitioning is to consider the connectivity of the underlying graph. The original algorithm [9] uses the following matching bound: let us consider some $p \in P_{R}$ and $q \in P_{B}$ with $\{p, q\} \in E$. We note that $p$ has a red edge adjacent (through its adjacent vertex in $R$ ), and $q$ has a blue edge adjacent. Clearly no matter what color we give $\{p, q\}$, we will have to cut one of $p$ and $q$.

We can improve upon this by finding a maximal set of such edges that are vertex-disjoint (this is necessary, since otherwise we could resolve two edges 
$\{p, q\}$ and $\left\{p, q^{\prime}\right\}$ just by cutting one vertex $\left.p\right)$. The relevant graph is just our bipartite graph $G(A)=(V, E)$ restricted to $P_{R} \cup P_{B} \subseteq V$, keeping only edges with one endpoint in $P_{R}$ and one in $P_{B}$. Bipartite matching is a classical problem we can solve in polynomial time. Then the size of the maximum matching is a lower bound on the number of vertices that still have to be cut by any extension of the current partial assignment.

This bound does not take full advantage of the connectivity of the graph since it only considers single edges that are adjacent to both a red and a blue edge (through $P_{R}$ and $P_{B}$ ). There is no need to restrict ourselves to single edges however, and this is especially clear when we consider the graph formulation of the problem. We now introduce a new flow bound.

Consider a path from $P_{R}$ to $P_{B}$ avoiding $R \cup B \cup C$, that is, a sequence of vertices $v_{1}, v_{2}, \ldots, v_{k}$ with $v_{i} \notin R \cup B \cup C$ for $1 \leq i \leq k$, and in particular $v_{1} \in P_{R}$ and $v_{k} \in P_{B}$, such that $\left\{v_{i}, v_{i+1}\right\} \in E$ for all $1 \leq i<k$. This corresponds to a series of intersecting rows and columns, the first of which contains a red nonzero (corresponding to the edge between $v_{1}$ and its neighbour in $R$ ) and the last a blue nonzero. One can see this in Figure 4. But then any extension of the current partial assignment that colors (assigns) all remaining vertices, must cut one of these vertices. If $v_{1}$ is not cut then it must be fully red, making $v_{2}$ partially red, and so on.

So if a single path between $P_{R}$ and $P_{B}$ implies that we have to cut at least one vertex, how do we extend this to multiple paths? Here we run in to the same issue as with the matching bound: if we have two paths that share a vertex, we can just cut that vertex to separate red and blue edges, for a cost of 1 .

Hence, to prove a lower bound of more than 1 we have to require that the paths are disjoint. In particular, the best lower bound we can hope for using this technique will be the maximum number of vertex-disjoint paths between $P_{R}$ and $P_{B}$. Note that these paths must be vertex-disjoint in $P_{R}$ and $P_{B}$ as well, since those vertices may also still be cut. Alternatively, when we imagine $R$ and $B$ as a single vertex, we are looking for a maximum number of paths from $R$ to $B$ that are vertex-disjoint outside of $R$ and $B$. In fact, a theorem by Menger shows that this is actually exactly the size of the smallest vertex cut.

Theorem 3.1. (Menger [26]) Let $G$ be a finite undirected graph, and let $u$ and $v$ be two non-adjacent vertices in $G$, then the size of the smallest vertex cut separating $u$ and $v$ equals the maximum number of vertex-disjoint paths between $u$ and $v$.

Unfortunately, the resulting bipartitioning may be very unbalanced. Intuitively, the flow bound might be small if there is a chokepoint between $R$ and $B$. However, the actual optimal bipartitioning might be much larger if this chokepoint is biased towards one of $R, B$. We would like to correct for this by adding another bound which considers the sizes of the neighbourhoods of $R$ and $B$ rather than their connectivity. This motivates the next class of bounds we introduce. 


\subsection{Packing Bounds}

The second bound used by Pelt and Bisseling [9] is a local packing bound. Let $E(R)$ denote all edges that are colored red by the current partial assignment (that is, all edges adjacent to a vertex in $R$ ). For each partially red vertex $p \in P_{R}$, let

$$
N_{\text {free }}(p)=\{e \in E \mid e \text { is adjacent to } p, e \notin E(R)\}
$$

denote all its adjacent unassigned edges. Note that all these edges are adjacent to a red edge (through $p$ ). If we do not want to cut any more vertices, we have to color all of $N_{\text {free }}(p)$ red. But if $|E(R)|+\sum_{p \in P_{R}}\left|N_{\text {free }}(p)\right|$ is greater than $(1+\epsilon) \frac{|E|}{2}$ this will lead to an unbalanced partition, and so we are forced to cut some of the vertices $p \in P_{R}$. Since we are looking for a lower bound, we can greedily take those vertices with largest $\left|N_{\text {free }}(p)\right|$ until the sum is small enough again.

Note that we implicitly assume all $N_{\text {free }}(p)$ are disjoint, so that we can assign their edges independently. Since $G(A)=(V, E)$ is bipartite, this is true if we consider each side of the bipartition separately (that is, the rows and the columns). We can then do the same for $P_{B}$, and add all unavoidable cuts together to get the packing bound.

As with the matching bound this bound only considers the direct neighbourhood of $R \cup B$ and leaves large sections of the graph unexamined. Here too, we use the fact that a path between a red edge and blue edge must have a cut somewhere in between to introduce a new, stronger packing bound. In particular we will look at whole subgraphs adjacent to $P_{R}$, rather than only at free edges incident to vertices in $P_{R}$. This bound is based on a similar approach taken by Delling and coworkers [14].

Definition 3.2. Given a graph $(V, E)$ with a partial assignment $R, B, C \subseteq V$, then an $R$-adjacent subgraph $\left(V^{\prime}, E^{\prime}\right)$ is a tuple of subsets $V^{\prime} \subseteq V, E^{\prime} \subseteq E$, satisfying the following properties:

(1) $V^{\prime}$ is disjoint from $R \cup B \cup C$.

(2) For any distinct $e_{1}, e_{2} \in E^{\prime}$ such that $u \in V$ is an endpoint of both $e_{1}$ and $e_{2}$, we have $u \in V^{\prime}$.

(3) $\left(V^{\prime}, E^{\prime}\right)$ is path-connected with respect to edges, i.e. for any $e_{1}, e_{2} \in E^{\prime}$ we can find $f_{1}, \ldots, f_{k} \in E^{\prime}$ pairwise incident, with $e_{1}=f_{1}$ and $f_{k}=e_{2}$.

(4) $\left(V^{\prime}, E^{\prime}\right)$ is adjacent to $R\left(V^{\prime} \cap P_{R} \neq \emptyset\right)$.

(5) All edges in $E^{\prime}$ are free in the partial partitioning $R, B, C$ (that is, no edges are adjacent to $R$ or $B$ ).

We can now use these subgraphs to find a lower bound on any extension of our partial assignment. Indeed, notice that for any edge in $E^{\prime}$ we can find a path (property 3 ) to an edge adjacent to $R$ (property 4 ) with all internal vertices in 
$V^{\prime}$ (property 2) and unassigned (property 1). Therefore coloring at least one of these edges blue requires us to cut at least one vertex in $V^{\prime}$.

Note that the definition intentionally does not require us to add both endpoints of an edge in $E^{\prime}$ to our vertex set $V^{\prime}$. This is because if we have an edge between our $R$-adjacent subgraph and $C$, this edge is still unassigned and we can therefore still find a path containing an internal vertex that has to be cut, in the event that this edge is colored blue.

We can now formulate a strategy to find a new, extended packing bound: we find a maximal collection of $R$-adjacent subgraphs $\left(V_{1}, E_{1}\right),\left(V_{2}, E_{2}\right), \ldots\left(V_{k}, E_{k}\right)$ that are pairwise disjoint. Denoting by $E(R)$ all edges incident to $R$, and noticing that all edges in $E_{i}$ are unassigned by the current partial assignment $R, B, C$ (property 5 ), then if we do not want to cut any more vertices and color all of the $R$-adjacent subgraphs red, this results in $|E(R)|+\sum_{i=1}^{k}\left|E_{i}\right|$ red edges. If this is larger than $(1+\epsilon) \frac{|E|}{2}$, any resulting partitioning would be unbalanced, and so we must cut some of the subgraphs. To find a lower bound we can again assume the ideal case where we cut the largest subgraphs (in terms of $\left|E_{i}\right|$ ) first, at a cost of one cut per subgraph. We can compute a similar quantity for $B$-adjacent subgraphs and add the results together for the extended packing bound.

\subsection{Implementation notes}

While the previous sections describe how to lower bound the volume given the existence of these specific paths and subgraphs, we are also interested in finding them. Additionally, there are various decisions one needs to make in the implementation process of a branch-and-bound algorithm, which we detail below in the interest of reproducibility.

\subsubsection{Branching strategy}

While we already specified that we branch on marking a row or column as red, blue, or cut, the order in which we select the rows and columns for branching could significantly affect the performance of the algorithm as this selection prescribes the order in which the entire search space is traversed.

Intuitively, it makes sense to branch on rows and columns with more free nonzeros, since their assignment affects the balance of the bipartitioning the most, and their high connectivity suggests they may be useful as sources of paths in the Flow Bound or subgraphs in the Extended Packing Bound. Thus, at each step we select for branching a row or column $u$ with a maximal number of unassigned nonzeros, breaking ties arbitrarily. Additionally, since the goal is to cut as few rows and columns as possible, we traverse the 'cut' subtree last, and since the goal is to balance the bipartition, we traverse first the subtree that assigns $u$ to the smallest side in the bipartition. We note that the previous program, MondriaanOpt, employs the same ordering strategy. As a remark, we refer to the paper by Mumcuyan et. al. 23] who show other branching strategies can be faster, and learn to predict the optimal strategy based on 
matrix statistics. It turns out that the strategy that performs best on average is exactly the one we implemented (branching on 'cut' vertices last and ordering by number of nonzeros).

\subsubsection{Initial upper bound}

To correctly prune, our branch-and-bound algorithm needs an upper bound to compare its lower bound against. Before we have found our first feasible solution we could use the trivial $\min (m, n)+1$ upper bound. Although this upper bound is in some sense tight (consider an odd square matrix with only one zero), for sparse matrices it is usually quite bad and forces our algorithm to consider many suboptimal solutions before arriving at better ones.

Instead, we would like to run the algorithm with an upper bound as tight as possible. Hence, we use a technique common in branch-and-bound algorithms where we run our algorithm with an initial (strict) upper bound of $U_{1}=1$, and rerun with $U_{i+1}=\left\lceil\frac{5}{4} U_{i}\right\rceil$ until we have found a solution. One may interpret this search technique as a variation of Iterative Deepening $A^{*}$, considering volume instead of path length.

\subsubsection{Implementing the flow bound}

Finding a maximal set of vertex-disjoint paths is a classical maximum flow problem. We can solve it by duplicating each vertex, and connecting the resulting vertex pairs with a capacity 1 edge to enforce that every vertex be used only once. A thorough exposition of this may be found in the book by Cormen et al. [27, chapter 26].

To leave as many edges for the packing bound as possible, we compute the maximum flow using shortest paths [28]. Finally, while the maximum flow problem may be solved in polynomial time, it still requires computation over the entire graph, which may slow down our algorithm for large matrices. Instead, we reuse the flow from the previous step in the branch-and-bound algorithm. Recomputing the flow then involves finding augmenting paths involving the modified vertex, which speeds up computation considerably especially when this subgraph is small, lower in the search tree.

\subsubsection{Extended Packing Bound}

The quality of this lower bound is highly dependent on the relative sizes of the subgraphs. Ideally, we would like the size of the largest subgraphs to be as small as possible, so we are forced to cut many of them to balance the partitioning. Thus, we start a depth-first search from all vertices in $R$ simultaneously, updating each of the corresponding search trees one by one (cycling through them using, for example, a queue) until all searches have terminated. 


\subsubsection{Combining bounds}

A priori, the flow and extended packing bound conflict with each other, so if we compute both we would be relegated to taking the maximum of the two (and then adding $|C|$ ). However we can first compute the flow bound, remove the set of paths it found from the graph, and then run the packing bound on the remainder of the graph. Then the packing bound will only use edges not used by the flow bound and we can add the two bounds together.

Additionally while the combined flow and extended packing bound together should give the strongest lower bound, they are also the most expensive to compute. Therefore we also compute the weaker, local packing bound [9], as the overhead to compute this is negligible. In particular, we do this computation incrementally: if this local packing bound on its own already indicates this subtree should be pruned, we do so without computing any other bounds. Then we compute the flow bound and ask the same question, and then finally the extended packing bound.

\section{Experimental results}

We implemented the branch-and-bound algorithm from Section 3 in our new program MP 3 , The implementation was done in $\mathrm{C}++14$, and the final program was compiled with GNU GCC Version 7.1.0 with the -02 flag. The program was written sequentially, but as a branch-and-bound algorithm it can easily be parallelized in the future.

To test the capabilities of the new exact matrix bipartitioner MP and to compare it with MondriaanOpt, we performed numerical experiments on a subset of small and medium-sized test matrices from the SuiteSparse Matrix Collection (formerly known as the University of Florida Sparse Matrix Collection [10]). We chose as test set the subset of all sparse matrices with at most 100,000 nonzeros, which contains 1602 matrices 4 . We chose a value of $\epsilon=0.03$ in Equation (2) which is a common value in the literature allowing a trade-off between load imbalance and communication volume. To keep the total CPU time used within reasonable bounds, we allotted a maximum of 24 hours of CPU time to each partitioning run.

All computations were carried out on thin nodes with 24 cores of the Dutch national supercomputer Cartesius at SURFsara in Amsterdam, with a core clock speed of $2.4 \mathrm{GHz}$ (for Intel Ivy Bridge E5-2695 v2 CPUs) or $2.6 \mathrm{GHz}$ (for Haswell Bridge E5-2690 v3 CPUs). The memory for each thin node is 64 GB. Each batch of 24 jobs is assigned to a node by a runtime scheduler, which may lead to different types of CPUs being used, causing a slight inconsistency in our timings. The scheduler carried out all MP runs on the slower Ivy Bridge nodes and all MondriaanOpt nodes on the faster Haswell nodes. Since this distribution favors

\footnotetext{
${ }^{3} \mathrm{MP}$ is available from https://github.com/TimonKnigge/matrix-partitioner

${ }^{4}$ After having removed five duplicate matrices: Pothen/barth, Pothen/barth4, Meszaros/fxm3_6, Boeing/nasa1824, Pajek/football.

${ }^{5}$ Retrieved September 2018.
} 
MondriaanOpt, we provide all results and runtimes uncorrected for this fact. For the sake of completeness, we performed calibration runs for the 40 longest running matrices using MP on both types of nodes of the Cartesius computer, and based on the geometric mean for this set, we found that the Haswell nodes are a factor of $\alpha=1.1782$ times faster than the Ivy Bridge nodes.

As a result of our experiments, we may divide the matrices into three groups: (i) a group of 368 matrices which could be solved by both programs, MP and MondriaanOpt. We use these matrices to compare the speed of the two programs and to verify their correctness; (ii) a group of 471 matrices which could only be solved by MP; (iii) a group of 763 matrices which could not be solved by either program. All matrices that could be solved by MondriaanOpt within 24 hours could also be solved by MP within 24 hours.

For the 368 matrices that could be solved by both programs, all optimal volumes computed are identical for the two programs, which we take as an independent mutual confirmation of their correctness. We have taken great care in developing the programs to make them understandable and to document them well, to support our claim that they compute exact, optimal solutions. Both programs are available as open-source software and are open to inspection for correctness. The two programs do not necessarily compute the same solution, as there may be several optimal solutions. The optimal volume, however, is of course unique.

Of the 368 matrices solved by both programs, MP is faster than MondriaanOpt in 306 cases ( $83 \%$ of the cases). In 25 cases $(6.7 \%)$, it performs equally well, of which 22 cases with both programs needing exactly 1 second (our clock resolution), and having a volume of 0 . In 37 cases (10\%), MondriaanOpt is faster, of which 31 cases with volume 0 . For volume 0 , the sparse matrix can be split into several connected components (when viewed as a graph) of suitable sizes. This situation is easy to handle and it is quickly discovered by both programs. In general, there is a tradeoff where the time spent computing sharper lower bounds should be compensated for by a sufficient reduction in the size of the branch-and-bound tree. In our situation this appears to be the case in a large majority of test matrices.

We synthesize the results of our experiments in Figure 5 below. With the exception of two matrices, mhd4800b (29574s) and mhd3200b (7977s), all matrices solved by MondriaanOpt in a day could be solved by MP in an hour, all but nine of them in fifteen minutes, and all but twenty in two minutes. The geometric average of the ratio $T_{\mathrm{MP}} / T_{\mathrm{Opt}}$ between the time of MP and the time of MondriaanOpt is 0.0855 . This average is based on 286 matrices that could be solved by both programs and for which $T_{\mathrm{MP}}, T_{\mathrm{Opt}} \geq 1$ second. This means that on average, MP is more than ten times faster than MondriaanOpt.

Table 1 shows the hardest (longest running) cases that MP could still solve within our self-imposed time limit. These are in fact the matrices that take between 3 and 24 hours to be solved. Note that there is no simple parameter that characterises the most difficult matrices for bipartitioning. Still we can say that the hardest solvable matrices usually have a communication volume of 10 or more, with the exception of the matrix mhd4800b, which has a low volume 

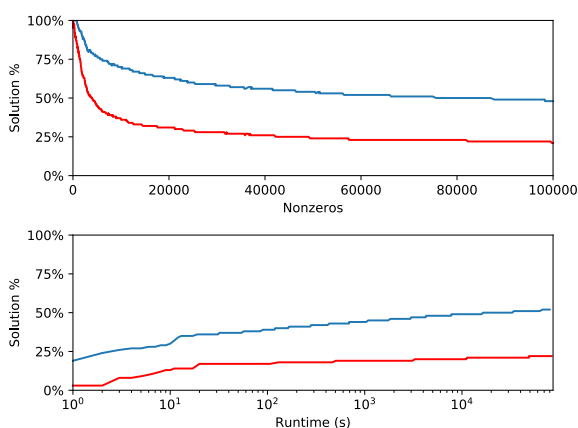

Figure 5: In both plots, the blue graph is for MP, and red for MondriaanOpt. The top plot shows for a given number of nonzeros $N$, what percentage of matrices with at most $N$ nonzeros were solved in the alloted time. The bottom plot shows for a given time in seconds $t$, the percentage of matrices in the whole test set that was solved in $t$ seconds. Note the logscale for $t$.

of 2. Furthermore, they also have at least 1000 nonzeros, with two exceptions, ch4-4-b2 and GD97_a. This particular top-40 set is our challenge to future exact partitioners.

In addition to the literature standard of $\epsilon=0.03$, we ran this top 40 of matrices for $\epsilon=0$ and $\epsilon=0.1$ as well, to see what the effect of the choice of $\epsilon$ might have on these hard matrices. Results are also found in Table 1 . We see that in many cases, the volume changes only slightly or not at all. There are several notable exceptions however, where the larger imbalance allows for lower volumes which can also be found faster, for example for the iiasa matrix. The comparative runtimes vary much more. Increasing $\epsilon$ will grow the size of the solution space since more partitionings now satisfy the load balancing constraint. Conversely, this may allow us to find lower volume solutions that we can use to prune larger parts of the search tree. Which of these effects is stronger depends on the matrix, even in a non-linear fashion as can be seen from the results of $1 p_{\text {p_grow2 } 22}$ where the choice of $\epsilon=0.03$ appears especially unfortunat 6 .

To emphasize the potential of MP as a benchmark for heuristic solutions, we applied Mondriaan 4.2.1 to the 839 optimally solved matrices. We used both the underlying hypergraph partitioner of Mondriaan as well as $\mathrm{PaToH}$ 3.2 , and used the fine-grain strategy (with and without iterative refinement) and medium-grain strategy (always uses iterative refinement). For all other options we relied on the Mondriaan defaults. The average runtime (arithmetic mean) and optimality ratio (geometric mean, after removing volume- 0 matrices, at which point 726 matrices remain) may be found in Table 2. Furthermore, Figure 6 contains a performance plot of the six configurations. To answer the

\footnotetext{
${ }^{6}$ Do note the selection bias here. Among the matrices that are very time consuming to solve for $\epsilon=0.1$ we might similarly find a matrix solved very rapidly for $\epsilon=0.03$.
} 
Table 1: The top-40 of matrices with the longest computation time needed by the matrix partitioner MP for $\epsilon=0.03$. Given are the matrix name, number of rows, columns, and nonzeros, and for each $\epsilon \in\{0,0.03,0.1\}$ the optimal communication volume and CPU time (in s) needed for computing an optimal solution. In case a matrix was not solved within the time limit of 24 hours, a dash $(-)$ appears. Of these matrices, MondriaanOpt only solved mhd4800b for $\epsilon=0.03$ (in 55001s).

\begin{tabular}{|c|c|c|c|c|c|c|c|c|c|}
\hline \multirow[t]{2}{*}{ Name } & \multirow[t]{2}{*}{$m$} & \multirow[t]{2}{*}{$n$} & \multirow[t]{2}{*}{$n z$} & \multicolumn{2}{|c|}{$\epsilon=0$} & \multicolumn{2}{|c|}{$\epsilon=0.03$} & \multicolumn{2}{|c|}{$\epsilon=0.1$} \\
\hline & & & & $V$ & $T$ & 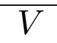 & $T$ & $V$ & $T$ \\
\hline$c-28$ & 4598 & 4598 & 30590 & 10 & 13275 & 10 & 11470 & 10 & 5494 \\
\hline mhd1280a & 1280 & 1280 & 47906 & - & - & 44 & 12375 & - & - \\
\hline bp_1000 & 822 & 822 & 4661 & 35 & 4058 & 35 & 13945 & - & - \\
\hline reorientation_4 & 2717 & 2717 & 33630 & - & - & 14 & 14680 & 14 & 28025 \\
\hline DK01R & 903 & 903 & 11766 & - & - & 20 & 15070 & 20 & 8527 \\
\hline west0479 & 479 & 479 & 1910 & 33 & 20982 & 33 & 15156 & 33 & 46986 \\
\hline $\operatorname{ch} 4-4-b 2$ & 96 & 72 & 288 & 24 & 23469 & 24 & 15955 & 24 & 39888 \\
\hline celegansneural & 294 & 270 & 2345 & 58 & 10142 & 57 & 16203 & - & - \\
\hline 1p_stocfor3 & 16675 & 23541 & 76473 & 14 & 13757 & 14 & 18138 & 14 & 37751 \\
\hline bayer 02 & 13935 & 13935 & 63679 & 28 & 18643 & 27 & 18832 & 27 & 30701 \\
\hline circuit204 & 1020 & 1020 & 5883 & - & - & 41 & 19078 & 40 & 35546 \\
\hline orbitRaising_4 & 915 & 915 & 7790 & - & - & 16 & 19405 & 8 & 14 \\
\hline GD97_a & 84 & 84 & 332 & 24 & 17958 & 24 & 20024 & 24 & 33897 \\
\hline lp_modszk1 & 686 & 1620 & 3168 & 34 & 60417 & 34 & 20768 & 34 & 45698 \\
\hline $\operatorname{mhd} 4800 \mathrm{~b}$ & 4800 & 4800 & 27520 & 2 & 23728 & 2 & 23298 & 0 & 12 \\
\hline Hamrle2 & 5952 & 5952 & 22162 & 16 & 13270 & 16 & 24322 & 16 & 6156 \\
\hline dynamicSoaringProblem_4 & 3191 & 3191 & 36516 & - & - & 22 & 24533 & 22 & 42353 \\
\hline pcb1000 & 1565 & 2820 & 20463 & 41 & 37923 & 40 & 24601 & 40 & 35283 \\
\hline lp_grow22 & 440 & 946 & 8252 & 20 & 340 & 20 & 25148 & 20 & 34 \\
\hline kineticBatchReactor_5 & 7641 & 7641 & 80767 & - & - & 18 & 26139 & 18 & 42805 \\
\hline can_256 & 256 & 256 & 2916 & 44 & 4193 & 43 & 28300 & 40 & 29983 \\
\hline qiulp & 1192 & 1900 & 4492 & - & - & 40 & 30445 & - & - \\
\hline ex21 & 656 & 656 & 19144 & - & - & 62 & 31311 & - & - \\
\hline lp_bnl1 & 642 & 1586 & 5532 & - & - & 47 & 31663 & - & - \\
\hline$c-29$ & 5033 & 5033 & 43731 & - & - & 28 & 32127 & - & - \\
\hline$f s_{-} 541 \_1$ & 541 & 541 & 4285 & 37 & 23259 & 37 & 33046 & - & - \\
\hline fs_541_4 & 541 & 541 & 4285 & 37 & 23247 & 37 & 33181 & - & - \\
\hline fs_541_2 & 541 & 541 & 4285 & 37 & 23529 & 37 & 33264 & - & - \\
\hline fs_541_3 & 541 & 541 & 4285 & 37 & 23303 & 37 & 33377 & - & - \\
\hline bp_600 & 822 & 822 & 4172 & - & - & 33 & 34244 & - & - \\
\hline model1 & 362 & 798 & 3028 & 47 & 17281 & 46 & 37081 & - & - \\
\hline kineticBatchReactor_9 & 8115 & 8115 & 86183 & - & - & 18 & 44655 & - & - \\
\hline kineticBatchReactor_4 & 7105 & 7105 & 74869 & - & - & 18 & 45586 & - & - \\
\hline de063157 & 936 & 1656 & 5119 & 36 & 8463 & 36 & 46148 & - & - \\
\hline ncvxqp9 & 16554 & 16554 & 54040 & 30 & 33325 & 30 & 46556 & 30 & 64409 \\
\hline 1p_sctap2 & 1090 & 2500 & 7334 & 41 & 29014 & 40 & 57920 & - & - \\
\hline iiasa & 669 & 3639 & 7317 & 14 & 11410 & 14 & 64331 & 6 & 3 \\
\hline can_229 & $229^{2}$ & 229 & 1777 & 38 & 42965 & 38 & 65317 & - & - \\
\hline lp_pilot4 & 410 & 1123 & 5264 & - & - & 47 & 68010 & 44 & 688 \\
\hline lpi_pilot4i & 410 & 1123 & 5264 & - & - & 47 & 70176 & 44 & 698 \\
\hline
\end{tabular}


question from the introduction 'how good are the current methods?', we note that the geometric average achieved by the combination of Mondriaan mediumgrain with iterative refinement and the $\mathrm{PaToH}$ bipartitioner is only $10 \%$ above the optimal attainable value, meaning that the heuristic partitioning is already very close to what can optimally be achieved.

\begin{tabular}{lcc}
\hline Partitioner & Runtime (in s) & Optimality ratio \\
\hline \hline Mondriaan FG & 0.051452 & 1.63409 \\
Mondriaan FG+IR & 0.053945 & 1.53390 \\
Mondriaan MG & 0.029856 & 1.46326 \\
PaToH FG & 0.013915 & 1.18534 \\
PaToH FG +IR & 0.015162 & 1.16161 \\
PaToH MG & 0.009188 & 1.10145 \\
\hline
\end{tabular}

Table 2: Results summary heuristic partitioners.



Figure 6: For a given ratio $r \geq 1$, we show what percentage of the 839 matrices were solved within a factor $r$ of the optimal solution found by MP, for Mondriaan fine-grain, Mondriaan fine-grain with iterative refinement, Mondriaan medium-grain, PaToH fine-grain, PaToH fine-grain with iterative refinement and finally $\mathbf{P a T o H}$ medium-grain. For matrices whose optimal volume was 0 , we used the convention that $x / 0=1$ when $x=0$ and $\infty$ if $x \geq 1$.

\section{Conclusions and future work}

In this work, we have expanded our data base of 356 optimally bipartitioned sparse matrices to 839 matrices, by developing a new flow-based bound and a stronger packing bound for our previous branch-and-bound algorithm [9]. We implemented this bound in a new matrix partitioner, MP, which has the same functionality as the previous partitioner MondriaanOpt. We are now able to bipartition $96.8 \%$ of the sparse matrices with at most 1000 nonzeros from the SuiteSparse collection [10] to optimality, reaching the exact minimum communication volume for a given load imbalance $\epsilon=0.03$. For matrices with less 
than 10,000 nonzeros, we are successful in $72.8 \%$ of the cases, and for matrices with less than 100,000 nonzeros still in 52.3\%. The new partitioner MP is more than ten times faster than MondriaanOpt for problems that both partitioners can solve, and, more importantly, enables us to solve many more partitioning problems.

In the near future, we intend to apply the new partitioner also to selected problems that we could not solve within our imposed limit of 24 hours. Looking already beyond the horizon, the smallest (by nonzeros) matrix that MP could not solve within our limit of one day is cage6. We partitioned this matrix using $\mathrm{MP}$ in $283,316 \mathrm{~s}$ (over 3 days) on a laptop computer with an Intel i7-8550U $1.8 \mathrm{GHz}$ CPU. By comparison, one of the authors ran MondriaanOpt for three months on this instance without solving it. The result of the 3 -day calculation is shown in Figure 7 .

In this paper, we also gave a proof of the $\mathcal{N} \mathcal{P}$-completeness of $\epsilon$-balanced sparse matrix bipartitioning. This result may hardly be surprising, as graph partitioning and hypergraph partitioning are both known to be $\mathcal{N} \mathcal{P}$-complete. Still, this problem is a very specific instance of hypergraph partitioning, and it is a motivation for developing good heuristic partitioners to know that solving the problem optimally by an exact algorithm is intractable. It is our hope that the expanded data base of optimally bipartitioned sparse matrices will be used in practice to benchmark the quality of the bipartitioning kernel of such heuristic partitioners.

\section{Acknowledgements}

We thank Oded Schwartz for helpful discussions on $\mathcal{N} \mathcal{P}$-completeness. The computations of this paper on the Dutch national supercomputer Cartesius at SURFsara in Amsterdam were carried out under grant SH-349-15 from The Netherlands Organisation for Scientific Research (NWO).

\section{References}

[1] G. Karypis, V. Kumar, Multilevel $k$-way hypergraph partitioning, in: Proceedings 36th ACM/IEEE Conference on Design Automation, ACM Press, New York, 1999, pp. 343-348. doi:doi.org/10.1145/309847.309954.

[2] Ü. V. Çatalyürek, C. Aykanat, Hypergraph-partitioning-based decomposition for parallel sparse-matrix vector multiplication, IEEE Transactions on Parallel and Distributed Systems 10 (7) (1999) 673-693. doi:10.1109/71. 780863.

[3] B. Vastenhouw, R. H. Bisseling, A two-dimensional data distribution method for parallel sparse matrix-vector multiplication, SIAM Review 47 (1) (2005) 67-95. doi:10.1137/S0036144502409019. 


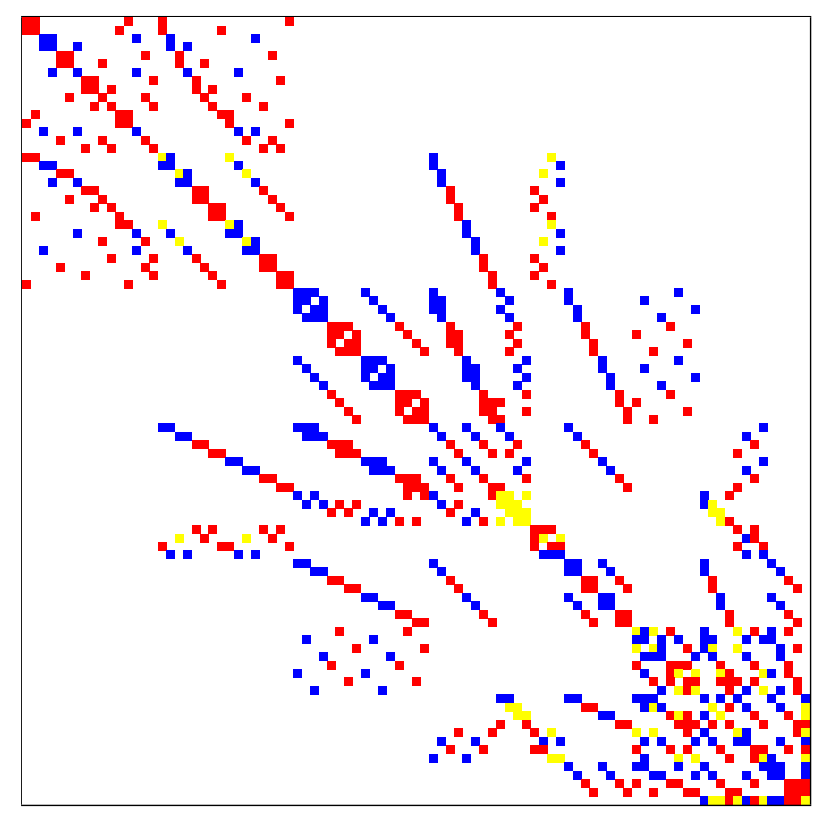

Figure 7: Bipartitioning of the $93 \times 93$ matrix cage 6 with 785 nonzeros. The minimum communication volume for an allowed imbalance of $\epsilon=0.03$ equals $V=38$. The 397 red nonzeros are assigned to one part, the 316 blue nonzeros to the other part, and the 72 yellow nonzeros can be freely assigned to any part without affecting the communication volume, because both their row and their column is already cut. We can color these free nonzeros blue to improve the load balance, giving 397 red and 388 blue nonzeros, corresponding to an achieved imbalance of about $\epsilon^{\prime}=0.01$.

[4] Y. Akhremtsev, T. Heuer, P. Sanders, S. Schlag, Engineering a direct $k$ way hypergraph partitioning algorithm, in: Proceedings of the Nineteenth Workshop on Algorithm Engineering and Experiments (ALENEX), 2017, pp. 28-42. doi:10.1137/1.9781611974768.3

[5] A. Trifunović, W. J. Knottenbelt, Parallel multilevel algorithms for hypergraph partitioning, Journal of Parallel and Distributed Computing 68 (5) (2008) 563-581. doi:10.1016/j.jpdc.2007.11.002.

[6] K. D. Devine, E. G. Boman, R. Heaphy, R. H. Bisseling, U. V. Catalyurek, Parallel hypergraph partitioning for scientific computing, in: Proceedings IEEE International Parallel and Distributed Processing Symposium 2006, IEEE Press, 2006, p. 102. doi:10.1109/IPDPS.2006.1639359.

[7] Ü. V. Çatalyürek, C. Aykanat, A fine-grain hypergraph model for 2D decomposition of sparse matrices, in: Proceedings IEEE International Parallel and Distributed Processing Symposium 2001, IEEE Press, 2001, p. 118. doi:10.1109/IPDPS.2001.925093. 
[8] D. M. Pelt, R. H. Bisseling, A medium-grain method for fast 2D bipartitioning of sparse matrices, in: Proceedings IEEE International Parallel and Distributed Processing Symposium 2014, IEEE Press, 2014, pp. 529-539. doi:10.1109/IPDPS.2014.62

[9] D. M. Pelt, R. H. Bisseling, An exact algorithm for sparse matrix bipartitioning, Journal of Parallel and Distributed Computing 85 (2015) 79-90. doi:10.1016/j.jpdc.2015.06.005.

[10] T. A. Davis, Y. Hu, The University of Florida sparse matrix collection, ACM Transactions on Mathematical Software 38 (1) (2011) 1:1-1:25. doi : $10.1145 / 2049662.2049663$.

[11] M. R. Garey, D. S. Johnson, Computers and Intractability; A Guide to the Theory of $\mathcal{N} \mathcal{P}$-Completeness, W. H. Freeman \& Co., New York, NY, USA, 1979.

[12] T. N. Bui, C. Jones, Finding good approximate vertex and edge partitions is NP-hard, Information Processing Letters 42 (3) (1992) 153-159. doi: 10.1016/0020-0190(92)90140-Q

[13] T. Lengauer, Combinatorial algorithms for integrated circuit layout, John Wiley and Sons, Chichester, UK, 1990. doi:10.1007/ 978-3-322-92106-2.

[14] D. Delling, D. Fleischman, A. V. Goldberg, I. Razenshteyn, R. F. Werneck, An exact combinatorial algorithm for minimum graph bisection, Mathematical Programming (2014) 1-42doi:10.1007/s10107-014-0811-z.

[15] O. Selvitopi, S. Acer, C. Aykanat, A recursive hypergraph bipartitioning framework for reducing bandwidth and latency costs simultaneously, IEEE Transactions on Parallel and Distributed Systems 28 (2) (2017) 345-358. doi:10.1109/TPDS.2016.2577024.

[16] S. E. Karisch, F. Rendl, J. Clausen, Solving graph bisection problems with semidefinite programming, INFORMS Journal on Computing 12 (3) (2000) 177-191. doi:10.1287/ijoc.12.3.177.12637.

[17] N. Sensen, Lower bounds and exact algorithms for the graph partitioning problem using multicommodity flows, in: Algorithms - Proceedings ESA 2001, Vol. 2161 of Lecture Notes in Computer Science, Springer, 2001, pp. 391-403. doi:10.1007/3-540-44676-1_33.

[18] A. Felner, Finding optimal solutions to the graph partitioning problem with heuristic search, Annals of Mathematics and Artificial Intelligence 45 (3-4) (2005) 293-322. doi:10.1007/s10472-005-9001-2.

[19] W. W. Hager, D. T. Phan, H. Zhang, An exact algorithm for graph partitioning, Mathematical Programming 137 (1-2) (2013) 531-556. doi: 10.1007/s10107-011-0503-x. 
[20] A. E. Caldwell, A. B. Kahng, I. L. Markov, Optimal partitioners and endcase placers for standard-cell layout, IEEE Trans. on CAD of Integrated Circuits and Systems 19 (11) (2000) 1304-1313. doi:10.1145/299996. 300032 .

[21] D. Kucar, S. Areibi, A. Vannelli, Hypergraph partitioning techniques, Dynamics of Continuous, Discrete and Impulsive Systems Series A: Mathematical Analysis 11 (2-3a) (2004) 339-367.

[22] R. H. Bisseling, J. Byrka, S. Cerav-Erbas, N. Gvozdenović, M. Lorenz, R. Pendavingh, C. Reeves, M. Röger, A. Verhoeven, Partitioning a call graph, in: Proceedings Study Group Mathematics with Industry 2005, Amsterdam, CWI, Amsterdam, 2005, pp. 95-107.

[23] A. Mumcuyan, B. Usta, K. Kaya, H. Yenigün, Optimally bipartitioning sparse matrices with reordering and parallelization, Concurrency and Computation: Practice and Experience 30 (21) (2018) e4687. doi:10.1002/ cpe.4687.

[24] M. R. Garey, D. S. Johnson, L. Stockmeyer, Some simplified $\mathcal{N} \mathcal{P}$-complete graph problems, Theoretical Computer Science 1 (3) (1976) 237-267. doi : $10.1145 / 800119.803884$.

[25] D. Wagner, F. Wagner, Between min cut and graph bisection, in: International Symposium on Mathematical Foundations of Computer Science, Springer, 1993, pp. 744-750. doi:10.1007/3-540-57182-5_65.

[26] K. Menger, Zur allgemeinen Kurventheorie, Fundamenta Mathematicae 10 (1) (1927) 96-115.

[27] T. H. Cormen, C. E. Leiserson, R. L. Rivest, C. Stein, Introduction to algorithms, Vol. 6, MIT Press, Cambridge MA, 2001.

[28] E. A. Dinic, Algorithm for solution of a problem of maximum flow in networks with power estimation, in: Soviet Math. Doklady, Vol. 11, 1970, pp. $1277-1280$. 